\title{
Evaluation of plasma brain-derived neurotrophic factor levels and self-perceived cognitive impairment post-chemotherapy: a longitudinal study
}

Terence $\mathrm{Ng}^{1,2+}$, Ying Yun Lee ${ }^{1 \dagger}$, Jung-woo Chae ${ }^{1,2}$, Angie Hui Ling Yeo ${ }^{1}$, Maung Shwe ${ }^{1}$, Yan Xiang Gan², Raymond C. H. Ng ${ }^{3,4}$, Pat Pak Yan Chu ${ }^{5}$, Chiea Chuen Khor ${ }^{6}$, Han Kiat Ho and Alexandre Chan ${ }^{1,2,3^{*}}$

\begin{abstract}
Background: Preliminary evidence suggests that changes in plasma brain-derived neurotrophic factor (BDNF) levels may contribute to the occurrence of chemotherapy-associated cognitive impairment (CACl), and a previous study suggested that carriers of the BDNF Met homozygous genotype are protected from CACl.

Methods: This multicenter, prospective cohort study involved chemotherapy-receiving early-stage breast cancer (ESBC) patients. Self-perceived cognitive function was longitudinally assessed using the validated FACT-Cog (ver. 3) across three time points: Prior to chemotherapy ( $(\mathrm{T} 1)$, during chemotherapy $(\mathrm{T} 2)$, and at the end of chemotherapy (T3). Plasma BDNF levels were quantified using enzyme-linked immunosorbent assay. Genotyping was performed using Sanger Sequencing.

Results: A total of 51 chemotherapy-receiving ESBC patients (mean age: $52.6 \pm 9.5$ years) were recruited, and 11 patients (21.6\%) reported subjective cognitive impairment post-chemotherapy. Overall, there was a reduction in median plasma BDNF levels over time (T1: $5423.0 \mathrm{pg} / \mathrm{ml} ; \mathrm{T} 2: 5313.6 \mathrm{pg} / \mathrm{ml} ; \mathrm{T3}: 4050.3 \mathrm{pg} / \mathrm{ml} ; p<0.01)$. After adjusting for confounding factors, longitudinal analysis revealed that BDNF levels were associated with self-reported concentration deficit $(p=0.032)$. Carriers of $\mathrm{Val} / \mathrm{Nal}(p=0.011)$ and $\mathrm{Val} / \mathrm{Met}(p=0.003)$ BDNF genotypes demonstrated a significant reduction in plasma BDNF levels over time; however, plasma BDNF levels were similar across all time points among Met homozygous carriers $(p=0.107)$.
\end{abstract}

Conclusion: There was a statistically significant change in BDNF levels post-chemotherapy in ESBC patients, and plasma BDNF levels were associated with self-perceived concentration deficit in patients receiving chemotherapy.

Keywords: BDNF, Breast cancer, Cognition, Genetics, rs6265

\section{Background}

Chemotherapy-associated cognitive impairment (CACI) among breast cancer survivors has been widely reported [1]. Often termed as "chemobrain," cognitive changes are subtle, yet notable. Memory, attention, and executive

\footnotetext{
*Correspondence: phaac@nus.edu.sg

${ }^{\dagger}$ Equal contributors

'Department of Pharmacy, Faculty of Science, National University of Singapore, Block S4A, 18 Science Drive 4, Level 3, Singapore 117543, Singapore

${ }^{2}$ Department of Pharmacy, National Cancer Centre Singapore, Singapore, Singapore

Full list of author information is available at the end of the article
}

function are particularly susceptible to chemotherapyinduced changes, and these changes may adversely affect a patient's daily functioning and quality of life.

While numerous mechanisms to explain chemobrain have been postulated, including direct chemotherapyinduced toxicities, immunologic alterations, and neural repair insufficiencies, little is definitively known about its actual causes. Of the proposed mechanisms, it has been suggested that the brain-derived neurotrophic factor (BDNF) may be implicated in CACI. BDNF is a type of neurotrophin extensively distributed in the central nervous system, particularly in the prefrontal cortex and 
hippocampus [2-4]. Through its action on tropomyosinrelated kinase B receptors [5], BDNF plays an essential role in regulating synaptic plasticity, neuronal growth, and survival $[3,4,6]$. In particular, it has been noted for its involvement in neurotransmitter release and long-term potentiation (LTP) [3, 4]. Long-term potentiation is important to memory and learning, and the inhibition of LTP may result in hippocampal-dependent memory impairment [7].

Numerous studies have reported the possible role of BDNF in the pathogenesis of various cognitive disorders, such as Alzheimer's disease [3, 4, 6, 8, 9]. Low serum BDNF levels have been correlated with Alzheimer's disease and mild cognitive impairment, and high serum BDNF levels have been associated with better cognition in healthy older adults. Studies have also suggested that plasma BDNF reflects cortical BDNF signaling during learning in healthy adults [10]. Consistent with the increasing evidence for BDNF's role in cognition, studies have also noted the contribution of BDNF Val66Met polymorphism (rs6265) in cognitive function and various neuropsychiatric disorders [11]. Our research group recently revealed that rs6265 confers a protective effect against CACI in the early-stage breast cancer (ESBC) population [12]. However, the trajectory of plasma BDNF during chemotherapy and its relation with rs6265 remain unknown.

Given the possible implication of BDNF in cognition, it is worthwhile exploring the possible association of peripheral BDNF levels and cognitive function in the chemotherapy-receiving cancer population. Hence, this pilot study was designed to investigate the changes of plasma BDNF levels and self-perceived cognitive impairment in ESBC patients receiving chemotherapy. A secondary objective of this study was to investigate the differences in plasma BDNF levels between the rs6265 genotypes.

\section{Methods}

\section{Study design and participants}

This was a prospective cohort study conducted at the National Cancer Centre Singapore and KK Women's and Children's Hospital between November 2014 to December 2015. This study was conducted in accordance to the Declaration of Helsinki and approved by SingHealth Institutional Review Board (CIRB 2014/754/B). Patients were given informed consent before recruitment. The inclusion criteria of this study were: (1) age $\geq 21$ years, (2) understood English or Mandarin, (3) diagnosed with ESBC (stage I-IIIa), (4) scheduled for anthracycline- or taxane-based chemotherapy, and (5) had no prior history of chemotherapy and/or radiotherapy. Exclusion criteria include (1) incapable of giving informed consent, (2) symptomatically ill, (3) presence of neuropsychiatric disorders, and (4) presence of neurologic or immune-related conditions.

\section{Procedures}

At the point of recruitment, patients' demographics and medical information were obtained via electronic medical records and patient interviews. Patients' selfperceived cognitive function and behavioral symptoms were assessed using subjective assessment tools at three time points in approximately 6 -week intervals: baseline (T1), during (T2) and at the end of chemotherapy (T3). The assessments were conducted either in English or Chinese by trained personnel. Each session was approximately $45 \mathrm{~min}$. At each time point, $10 \mathrm{~mL}$ of whole blood was collected in a heparinized-tube and immediately centrifuged at $2500 \mathrm{rpm}$ for $10 \mathrm{~min}$. The plasma and buffy coat were stored at $-80^{\circ} \mathrm{C}$ until analysis.

\section{Assessment of self-perceived cognitive impairment}

The Functional Assessment of Cancer TherapyCognitive Function (FACT-Cog) version is a 37 -item questionnaire which evaluates patients' self-perceived cognitive decline within the past 7 days, and this tool has been validated for usage in our population [13]. Six domains of cognition (mental acuity, attention and concentration, memory, verbal fluency, functional interference and multitasking ability) are evaluated on a scale of 0 ("Never" or "Not at all") to 4 ("Several times a day" or "Very much"). Reverse scores from individual items are tallied to obtain the global FACT-Cog score. A higher score denotes better self-perceived cognitive function. The patients were classified as having self-perceived cognitive impairment if there was a reduction of at least 10.6 points in the global FACT-Cog score at $\mathrm{T} 2$ or $\mathrm{T} 3$ relative to the baseline value. For each cognitive subdomain, a 15\% decrease score reduction at $\mathrm{T} 2$ or $\mathrm{T} 3$ relative to the baseline value is considered as impairment. This classification has been utilized in other studies that evaluated selfperceived cognitive impairment in cancer patients $[12,14]$.

\section{Assessment of cancer-related fatigue}

Cancer-related fatigue was assessed using the Brief Fatigue Inventory (BFI) [15]. The BFI measures the severity of fatigue (a known confounder of cognition) at the point of assessment and in the past $24 \mathrm{~h}$, rated in a scale of 0 ("no fatigue") to 10 ("fatigue as bad as you can imagine"). BFI assesses whether fatigue interfere on daily activities in the past $24 \mathrm{~h}$, on a scale of 0 ("does not interfere") to 10 ("completely interferes"). Six aspects of interference were assessed: general activity, mood, walking ability, normal work, relations with other people and enjoyment of life.

\section{Assessment of anxiety}

Anxiety was assessed using the Beck Anxiety Inventory (BAI), and this tool has been validated for usage in our population [16]. The BAI measures the severity of anxiety, a known confounder of cognition, in the past month 
based on 21 items listing common anxiety symptoms, on a scale of 0 ("not at all") to 3 ("severe"). Scores from individual items were tallied to obtain a global score. A higher score denotes greater anxiety.

\section{Assessment of depression}

Depression was assessed using the Beck Depression Inventory (BDI) [17]. The BDI evaluates 21 symptoms of depression (14 cognitive-affective symptoms, 7 somatic symptoms) on a four-point intensity scale. Scores were tallied to a total score. A higher score reflects greater severity of depression.

\section{Plasma BDNF quantification}

Plasma BDNF levels were analyzed in duplicate using a commercial enzyme-linked immunosorbent assay (ELISA) kit (Biosensis ${ }^{\circ}$ BEK-2211-2P, Australia) according to the manufacturer's instructions. The kit detects $100 \%$ of the mature BDNF, with less than $7 \%$ cross-reactivity with the pro-form of BDNF. Brain-derived neurotrophic factor standards $(7.8-500.0 \mathrm{pg} / \mathrm{mL})$ were prepared via serial dilutions, and the plasma samples underwent dilution by a factor of 50. Brain-derived neurotrophic factor was quantified at $450 \mathrm{~nm}$ using a plate reader. The standard curve was constructed using a four-parameter logistic model using ElisaAnalysis.com (Leading Technology Group, Australia) software. An intra-assay coefficient of variance (CV) of less than $10 \%$ was considered to be acceptable.

\section{Genotyping}

Genomic DNA from the buffy coat was isolated using a QIAamp DNA Blood Mini Kit (Qiagen, Germany). The region containing the BDNF rs6265 polymorphism was amplified by polymerase chain reaction (PCR) using specific and optimized primers. The primers involved were: 5'-GGACTCTGGAGAGCGTGAA-3' (forward) and 5'-CGTGTACAAGTCTGCGTCCT-3 (reverse). Genotyping of the PCR products was performed by automated Sanger sequencing using a 3730xl DNA Analyzer (Applied Biosystems, United States). Samples were identified only by codes, and genotyping was blindly performed by AITbiotech without knowledge of the clinical outcomes.

\section{Statistical analysis}

All of the statistical analyses were performed using Stata Version 14 (StataCorp, 2015). Descriptive statistics were utilized to summarize the demographic and clinical characteristics of the patients. The Friedman test was utilized to evaluate changes in the plasma BDNF levels over time, and the post-hoc Wilcoxon signed-rank test was used for pair-wise comparisons between individual time points. Deviation of the genotypes from Hardy-Weinberg equilibrium was calculated using the chi-squared test with one degree of freedom.
To investigate the association between plasma BDNF levels and self-perceived cognitive function (overall and each of the six domains) over time, we created a generalized estimating equations (GEE) model. Selection of the appropriate correlation structures in the GEE model was conducted using the quasilikelihood under the independence model criterion, the structure exhibiting the smallest criterion was considered as the most desirable. Documented confounders of BDNF levels and self-perceived CACI (fatigue, anxiety, depression, age, body mass index (BMI) and BDNF genotypes) were included in the statistical model [12, 18].

Subgroup analyses were performed to determine the change in plasma BDNF levels after classifying for selfperceived cognitive impairment and the BDNF rs6265 genotypes. The Kruskal-Wallis test and Mann-Whitney $\mathrm{U}$ test were used to analyze cross-sectional differences in plasma BDNF levels between the genotypes and to analyze differences in plasma BDNF levels between cognitively impaired and non-impaired population. All of the statistical tests were two-sided, and $p<0.05$ was considered to correspond to statistical significance.

\section{Results \\ Demographics and clinical information}

The analysis included 51 ESBC patients with a mean age of $52.6 \pm 9.5$ years (Table 1 ). The patients were predominantly Chinese $(78.4 \%)$, and 44 patients $(86.2 \%)$ had completed at least secondary school. Thirty-two patients (62.8\%) were diagnosed with stage II breast cancer. All of the patients were ambulatory without activity restrictions. Twenty-nine patients (56.9\%) received anthracyclinebased chemotherapy, and 22 patients $(43.1 \%)$ received taxane-based chemotherapy. Using the Minimal Clinical Important Difference (MCID) of the FACT-Cog, 11 patients (21.6\%) were classified as manifesting self-perceived cognitive impairment.

Analysis of the behavioral symptoms revealed a statistical significant increase in median fatigue levels (0.67 at T1 vs. 1.67 at T3, $p<0.001)$. Median anxiety levels, as measured by BAI, increased from 0 at T1 to 6 at T3 $(p=0.040)$, while depression severity, as measured by BDI, increased from a median score of 3 at T1 to 6 at T3 $(p<0.001)$.

\section{Genotypes and allele frequencies}

Fifty patients were successfully genotyped for the BDNF rs6265 polymorphism. The genotype frequency did not deviate from the Hardy-Weinberg Equilibrium $\left(x^{2}=0.07\right.$, $p>0.05)$. The Val (52.0\%) and Met (48.0\%) allele frequency was approximately equivalent. (Table 2). 
Table 1 Demographics and clinical information of patients $(n=51)$

\begin{tabular}{|c|c|c|}
\hline \multicolumn{2}{|l|}{ Characteristics } & \multirow{2}{*}{$\begin{array}{l}n(\%) \\
52.6 \pm 9.5\end{array}$} \\
\hline Age (years, mean $\pm S D$ ) & & \\
\hline \multirow[t]{4}{*}{ Race } & Chinese & $40(78.4)$ \\
\hline & Malay & $4(7.8)$ \\
\hline & Indian & $6(11.8)$ \\
\hline & Other & $1(2.0)$ \\
\hline \multirow[t]{3}{*}{ Marital status } & Single & $8(15.7)$ \\
\hline & Married & $40(78.4)$ \\
\hline & Divorced & $3(5.9)$ \\
\hline \multirow[t]{5}{*}{ Education level } & None & $1(2.0)$ \\
\hline & Primary school & $6(11.8)$ \\
\hline & Secondary school & $22(43.1)$ \\
\hline & Pre-university & $7(13.7)$ \\
\hline & Graduate/postgraduate & $15(29.4)$ \\
\hline \multirow[t]{4}{*}{ Occupation } & Currently working & $36(70.6)$ \\
\hline & Currently not working & $13(25.4)$ \\
\hline & Retired & $1(2.0)$ \\
\hline & Long-term medical leave & $1(2.0)$ \\
\hline \multirow[t]{3}{*}{ Cancer diagnosis } & Stage 1 & $7(13.7)$ \\
\hline & Stage ॥ & $32(62.8)$ \\
\hline & Stage III & $12(23.5)$ \\
\hline ECOG Performance status & 0 & $51(100.0)$ \\
\hline \multirow[t]{2}{*}{ Menopausal status } & Pre-menopausal & $20(39.2)$ \\
\hline & Post-menopausal & $31(60.8)$ \\
\hline \multirow[t]{2}{*}{ Chemotherapy regimen } & Anthracycline-based & $29(56.9)$ \\
\hline & Taxane-based & $22(43.1)$ \\
\hline
\end{tabular}

Behavioral symptoms, median (IQR)

Baseline fatigue (BFI total score, out of 10$)$

$0.7(0.1,1.8)$

Baseline anxiety (BAI total score, out of 63)

$0.0(0.0,0.0)$

Baseline depression (BDI total score, out of 63)

$3.0(1.0,8.0)$

Abbreviations: $S D$ standard deviation, IQR interquartile range, ECOG Eastern Cooperative Oncology Group, BFI Brief Fatigue Inventory, BAl Beck Anxiety Inventory, $B D I$ Beck Depression Inventory

\section{Trajectory of plasma BDNF levels over time}

All of the plasma BDNF levels fell within the BDNF standard curve $(7.8-500.0 \mathrm{pg} / \mathrm{mL})$ after dilution. The range of BDNF levels detected with ELISA, after correcting for the dilution factor, was $538.6-23,218.7 \mathrm{pg} / \mathrm{mL}$. The mean intra-assay CV obtained was $4.9 \%$. There was a statistically significant difference in BDNF levels across the three time points (T1: 5423.0 vs. T2: 5313.6 vs. T3: $4050.3 \mathrm{pg} / \mathrm{mL} ; p<0.001)$, with a decreasing trend over time (Fig. 1a).

Among individuals who were cognitively impaired $(n=11)$, BDNF levels were statistically different over time (T1: 5423.0 vs. T2: 5823.2 vs. T3: $3095.7 \mathrm{pg} / \mathrm{mL} ; p=0.029$ ) (Fig. 1b). A post-hoc comparison of BDNF levels at $\mathrm{T} 3$ versus $\mathrm{T} 1$ was
Table 2 Genotype and allele frequencies of the BDNF Val66Met polymorphism $(N=50)^{a}$

\begin{tabular}{|c|c|c|c|c|c|}
\hline & \multicolumn{4}{|c|}{ Population, n (\%) } & \multirow{2}{*}{$\begin{array}{l}\text { Pooled } \\
\text { Asians, } \\
n(\%)\end{array}$} \\
\hline & & Malay & & & \\
\hline & $(n=40)$ & $(n=4)$ & $(n=5)$ & $(n=1)$ & $(n=50)$ \\
\hline \multicolumn{6}{|c|}{ Genotype frequency } \\
\hline GG (Val/Nal) & $11(27.5)$ & $1(25.0)$ & $2(40.0)$ & $0(0.0)$ & $14(28.0)$ \\
\hline GA (Val/Met) & $18(45.0)$ & $3(75.0)$ & $2(40.0)$ & $1(100.0)$ & $24(48.0)$ \\
\hline AA (Met/Met) & $11(27.5)$ & $0(0.0)$ & $1(20.0)$ & $0(0.0)$ & $12(24.0)$ \\
\hline \multicolumn{6}{|l|}{ Allele frequency } \\
\hline G (Val) allele & $40(50.0)$ & $5(62.5)$ & $6(60.0)$ & $1(50.0)$ & $52(52.0)$ \\
\hline A (Met) allele & $40(50.0)$ & $3(37.5)$ & $4(40.0)$ & $1(50.0)$ & $48(48.0)$ \\
\hline
\end{tabular}

${ }^{\mathrm{a} G e n o t y p e ~ d a t a ~ f o r ~ o n e ~ p a t i e n t ~ i s ~ n o t ~ a v a i l a b l e ~}$

${ }^{b}$ Others include 1 Filipino

statistically significant $(p=0.016)$. Similarly, non-impaired individuals $(n=40)$ experienced a significant reduction in BDNF levels across the three-time points $(p<0.001)$.

A comparison of plasma BDNF levels at baseline did not reveal statistically significant difference between selfperceived cognitively impaired $(5423.0 \mathrm{pg} / \mathrm{mL})$ and nonimpaired individuals $(5430.0 \mathrm{pg} / \mathrm{mL})(p=0.664)$. Similarly, plasma BDNF levels were similar between the impaired and non-impaired populations during chemotherapy $(5823.2 \mathrm{pg} / \mathrm{mL}$ vs. $5313.6 \mathrm{pg} / \mathrm{mL} ; p=0.309)$ and after chemotherapy (3095.7 vs. $4069.5 \mathrm{pg} / \mathrm{mL} ; p=0.336$ ).

\section{Associations between plasma BDNF levels and self- perceived $\mathrm{CACl}$}

After accounting for known confounders of selfreported cognitive impairment, the GEE model revealed that BDNF levels were found to be associated with self-perceived concentration deficit. (Table 3). BAI was associated with the proportion of overall self-perceived cognitively impaired individuals over time $(p<0.001)$. BAI was also associated with selfperceived mental acuity deficit $(p=0.001)$, selfperceived concentration deficit $(p=0.047)$, selfperceived memory deficit $(p=0.007)$, self-perceived verbal fluency interference $(p=0.001)$, self-perceived functional interference $(p=0.006)$ and self-perceived multitasking ability interference $(p=0.001)$, while BFI was associated with self-perceived concentration deficit $(p<0.001)$, self-perceived memory deficit $(p=0.009)$ and self-perceived functional interference $(p=0.040)$.

The BDNF Met risk allele was found to be associated with both overall self-perceived cognitive impairment $(p=0.041)$ and self-perceived concentration deficit $(p=0.043)$. There were no statistically significant associations between self-perceived cognitive disturbances with age and BMI. 


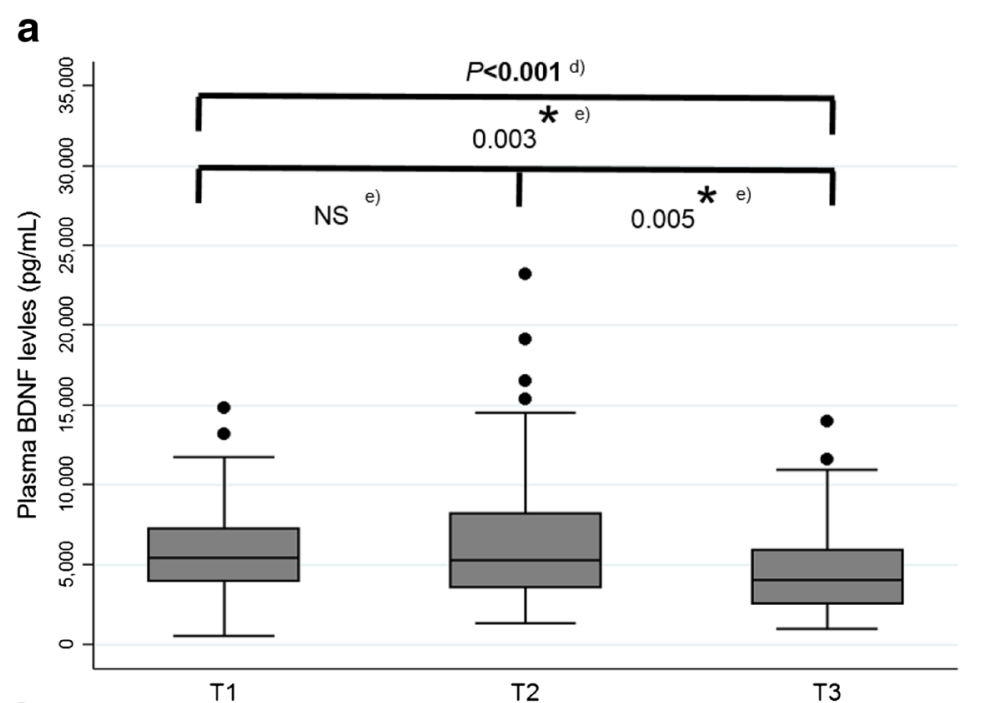

b
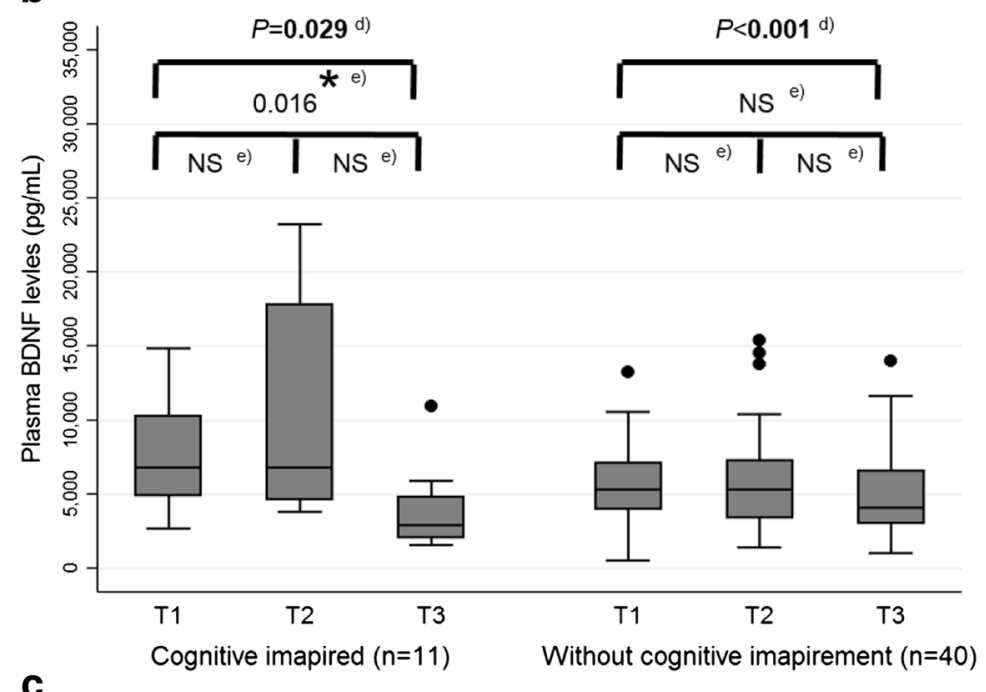

C

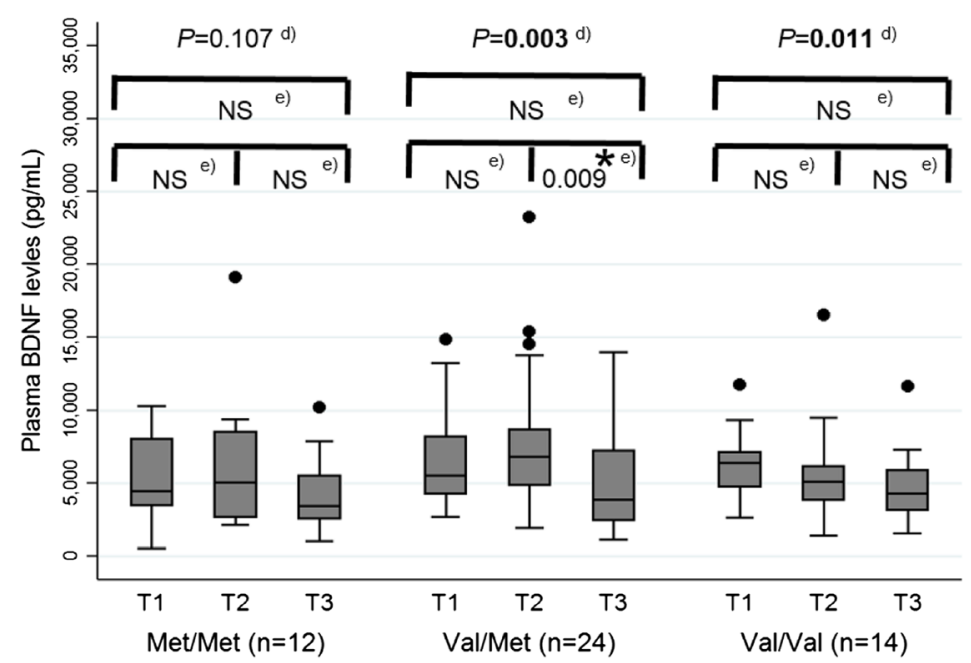

Fig. 1 Trajectory of plasma BDNF levels over time $(n=51)$. a Classified based on the entire patient pool; $\mathbf{b}$ classified based on overall cognition status; c classified based on BDNF rs6265 genotypes; $\mathbf{d}$ the Friedman test was used to evaluate changes in the plasma BDNF levels over time, e Post-hoc analysis was conducted using the Wilcoxon signed rank test for pair-wise comparisons. ${ }^{*} P$ values are significant if they are less than 0.0167 







\section{Change of plasma BDNF levels in relation to BDNF Val66Met polymorphism classification}

There was a statistically significant change in plasma BDNF levels over time among the Val/Val $(p=0.011)$ and $\mathrm{Val} / \mathrm{Met}$ genotype $(p=0.003)$; however, the change was not statistically significant among carriers of the Met/Met genotype ( $p=0.107)$ (Fig. 1c).

\section{Discussion}

Given the role of BDNF in mediating synaptic plasticity and neuronal growth in the hippocampus, studies have suggested BDNF's involvement in cognitive function, particularly in learning and memory $[3,4,6,7]$. This study sought to investigate the changes of plasma BDNF levels and self-perceived cognitive impairment in ESBC patients receiving chemotherapy. We have observed a statistically significant change in plasma BDNF levels over time post-chemotherapy, and such observation was relevant in both self-perceived cognitive impaired and non-impaired subgroups. To further elucidate the relationship between BDNF levels and self-perceived cognitive impairment, after adjusting for known confounders (including BFI, BAI, BDI, age, BMI and BDNF genotypes) of self-perceived cognitive impairment, our longitudinal model revealed that BDNF levels were associated with self-perceived concentration deficit. However, changes in BDNF levels were not observed among those who were Met homozygous carriers of the BDNF genotype.

Although we have observed an association between BDNF levels and self-perceived concentration deficit, the findings are not consistent with other published studies. Such inconsistencies could be attributed to differences in the study designs, disease populations, and cognitive assessment tools. Previous studies were mainly cross-sectional in nature, and they were conducted in non-cancerous populations. A number of longitudinal studies did not establish any associations between serum BDNF levels and cognitive decline in healthy older adults $[19,20]$. However, one study revealed that BDNF levels in the cerebrospinal fluid (CSF) were significantly associated with greater decline in cognitive function, specifically within the memory domain in healthy older adults [21]. This finding suggests that plasma BDNF levels may not be fully representative of the CSF BDNF levels in the brain. Although BDNF is able to cross the bloodbrain barrier [22], the degree to which plasma BDNF levels can represent CSF BDNF levels remains unclear $[23,24]$. Further investigations have to be conducted to draw a conclusion on the use of plasma BDNF levels as a surrogate marker for CSF BDNF levels.

Emerging studies have highlighted the contrasting roles of BDNF isoforms in cognition [25-28]. Biologically, BDNF is synthesized as a precursor known as proBDNF prior to cleavage into the mature BDNF (mBDNF) via furin intracellularly or matrix metalloproteinases (MMP) and plasmin extracellularly [26, 27, 29] (Fig. 2). ProBDNF is necessary for promoting the folding of the mature domain and for sorting BDNF into secretory vesicles. While mBDNF regulates neuronal




growth and survival, proBDNF preferentially binds to p75 neurotrophin receptor (p75NTR) to activate neuronal apoptotic pathways [27, 30]. In a sample of autistic patients, it was suggested that an imbalance in BDNF isoforms may have been a possible mechanism leading to autism [25]. High plasma BDNF levels may not indicate better cognition if proBDNF predominates over BDNF, which results in greater neuronal apoptosis. Another study revealed that the direction of BDNF regulation could be affected by mechanisms controlling the cleavage of proBDNF [31]. High-frequency stimulation was found to promote the release of proBDNF and tissue plasminogen activator; low-frequency stimulation only resulted in proBDNF release. However, this study did not evaluate the expressions levels of proBDNF. Future studies should evaluate the ratio between mBDNF and proBDNF in the context of CACI.

In this study, the Met risk allele is associated with both overall self-perceived cognitive impairment and self-perceived concentration deficit. This is in agreement with the literature that the BDNF Val66Met polymorphism (rs6265) may contribute to varying cognitive function. One study has suggested that the Met allele confers a protective effect against CACI in patients receiving chemotherapy, specifically in the areas of verbal fluency and multitasking ability [12]. The BDNF Val66Met polymorphism involves a non-synonymous single nucleotide polymorphism (SNP) that results in the substitution of Valine to Methionine at codon66 at the proBDNF region. As a result of this change, there is an impaired sorting of BDNF into secretory vesicles [11]. This impairment might lead to a reduction of proBDNF release, which is the major BDNF isoform present in secretory vesicles. Consequently, carriers of the Met allele would express lower baseline levels of proBDNF compared to the carriers of the Val allele. It is unknown, however, whether reduced level of proBDNF could affect mBDNF levels by other compensatory mechanisms. Further studies are required in order to elucidate the relationship between BDNF polymorphism and proBDNF expression in the CACI setting.

The strengths of this study include its prospective nature and the longitudinal assessment of cognitive function and behavioral symptoms. Since the manifestation of CACI is complex and can be influenced by multiple factors, documented confounders [12, 18] such as anxiety, fatigue and depression (using BAI, BFI and BDI) were also evaluated in this study. However, one major limitation was the relatively small sample size that subjects this study to Type 2 errors. A study with larger sample size is required to confirm this finding. This study also lacks an objective assessment of cognitive function using neuropsychological batteries, as recommended by the International Cognition and Cancer Task Force [32]. A larger sample size, as well as a replication of the findings of this study in an independent cohort at a different site deems important. Hence, future studies should validate the findings of this study.

\section{Conclusions}

In this pilot study, we have observed a statistically significant change in BDNF levels post-chemotherapy. After adjusting for potential confounders, a change in BDNF levels was associated with self-perceived concentration deficit. BDNF levels, however, remain similar over time among carriers of the Met homozygous carriers of the BDNF rs6265 polymorphism. Given the complexity of BDNF and cognitive function, additional studies taking into account proBDNF, MMP, and plasmin should be conducted in order to gain a better understanding of the contribution of the various BDNF isoforms to CACI.

\begin{abstract}
Abbreviations
BAl: Beck Anxiety Inventory; BDI: Beck Depression Inventory; BDNF: Brainderived neurotrophic factor; BFI: Brief Fatigue Inventory;

CACl: Chemotherapy-associated cognitive impairment; CSF: Cerebrospinal fluid; CV: Coefficient of variance; ELISA: Enzyme-linked immunosorbent assay; ESBC: Early-stage breast cancer; FACT-Cog: Functional Assessment of Cancer Therapy-Cognitive Function; LTP: Long-term potentiation; MMP: Matrix metalloproteinases; SNP: Single nucleotide polymorphism
\end{abstract}

\section{Acknowledgements \\ The authors acknowledge the contributions of all of the study participants. We also thank the Department of Pharmacy, National University of Singapore for providing support for this project. \\ Funding \\ This study was supported by research grants awarded by the National Medical Research Council Singapore (NMRC/CIRG/1386/2014). The funding body had no role in the design of the study and collection, analysis, and interpretation of data and in writing the manuscript.}

\section{Availability of data and materials}

The data set used is locked and stored in the pharmacy office at the National Cancer Centre Singapore where they can be accessed.

\section{Authors' contributions}

TN, YYL, HLAY, MS, YXG and AC are involved with the planning of the study. The study was conducted by all authors (TN, YYL, JWC, HLAY, MS, YXG, RN, PYPC, CCK, HHK, AC). Reporting of the work, as well as the approval of the final manuscript was conducted by all authors.

\section{Ethics approval and consent to participate}

Ethics approval by SingHealth Institutional Review Board was attained prior to the execution of the study. Patients have provided informed consent prior to enrollment in this study (CIRB 2014/754/B).

\section{Consent for publication}

All authors have provided consent for publication.

Competing interests

The authors declare that they have no competing interests. 


\section{Publisher's Note}

Springer Nature remains neutral with regard to jurisdictional claims in published maps and institutional affiliations.

\section{Author details \\ 'Department of Pharmacy, Faculty of Science, National University of Singapore, Block S4A, 18 Science Drive 4, Level 3, Singapore 117543, Singapore. ${ }^{2}$ Department of Pharmacy, National Cancer Centre Singapore, Singapore, Singapore. ${ }^{3}$ Duke-NUS Medical School Singapore, Singapore, Singapore. ${ }^{4}$ Division of Medical Oncology, National Cancer Centre Singapore, Singapore, Singapore. ${ }^{5}$ Singapore Cord Blood Bank, K.K. Women's and Children's Hospital, Singapore, Singapore. ${ }^{6}$ Genome Institute of Singapore, Singapore, Singapore.}

Received: 29 June 2016 Accepted: 29 November 2017 Published online: 19 December 2017

\section{References}

1. Cheung YT, Shwe M, Tan YP, Fan G, Ng R, Chan A. Cognitive changes in multiethnic Asian breast cancer patients: a focus group study. Ann Oncol. 2012;23(10):2547-52

2. Binder DK, Scharfman HE. Brain-derived neurotrophic factor. Growth Factors (Chur, Switzerland). 2004;22(3):123-31.

3. Teixeira AL, Barbosa IG, Diniz BS, Kummer A. Circulating levels of brainderived neurotrophic factor: correlation with mood, cognition and motor function. Biomark Med. 2010:4(6):871-87.

4. Zhang XY, Chen da C, Xiu MH, Haile CN, Luo X, Xu K, Zhang HP, Zuo L, Zhang $Z$, Zhang $X$, et al. Cognitive and serum BDNF correlates of BDNF Val66Met gene polymorphism in patients with schizophrenia and normal controls. Hum Genet. 2012;131(7):1187-95.

5. Adachi N, Numakawa T, Richards M, Nakajima S, Kunugi $H$. New insight in expression, transport, and secretion of brain-derived neurotrophic factor: implications in brain-related diseases. World J Biol Chem. 2014;5(4):409-28.

6. Gunstad J, Benitez A, Smith J, Glickman E, Spitznagel MB, Alexander T, Juvancic-Heltzel J, Murray L. Serum brain-derived neurotrophic factor is associated with cognitive function in healthy older adults. J Geriatr Psychiatry Neurol. 2008:21(3):166-70.

7. Morris RG, Anderson E, Lynch GS, Baudry M. Selective impairment of learning and blockade of long-term potentiation by an N-methyl-Daspartate receptor antagonist, AP5. Nature. 1986;319(6056):774-6.

8. Yu H, Zhang Z, Shi Y, Bai F, Xie C, Qian Y, Yuan Y, Deng L. Association study of the decreased serum BDNF concentrations in amnestic mild cognitive impairment and the Val66Met polymorphism in Chinese Han. J Clin Psychiatry. 2008:69(7):1104-11.

9. Shimada H, Makizako H, Doi T, Yoshida D, Tsutsumimoto K, Anan Y, Uemura K, Lee S, Park H, Suzuki T. A large, cross-sectional observational study of serum BDNF, cognitive function, and mild cognitive impairment in the elderly. Front Aging Neurosci. 2014;6:69.

10. Skilleter AJ, Weickert CS, Vercammen A, Lenroot R, Weickert TW. Peripheral BDNF: a candidate biomarker of healthy neural activity during learning is disrupted in schizophrenia. Psychol Med. 2015:45(4):841-54

11. Gratacos M, Gonzalez JR, Mercader JM, de Cid R, Urretavizcaya M, Estivill X Brain-derived neurotrophic factor Val66Met and psychiatric disorders: meta-analysis of case-control studies confirm association to substancerelated disorders, eating disorders, and schizophrenia. Biol Psychiatry. 2007;61(7):911-22

12. Ng T, Teo SM, Yeo HL, Shwe M, Gan YX, Cheung YT, Foo KM, Cham MT, Lee JA Tan YP, et al. Brain-derived neurotrophic factor genetic polymorphism (rs6265) is protective against chemotherapy-associated cognitive impairment in patients with early-stage breast cancer. Neuro-Oncology. 2016;18(2):244-51.

13. Cheung YT, Lim SR, Shwe M, Tan YP, Chan A. Psychometric properties and measurement equivalence of the English and Chinese versions of the functional assessment of cancer therapy-cognitive in Asian patients with breast cancer. Value Health. 2013;16(6):1001-13.

14. Cheung YT, Foo YL, Shwe M, Tan YP, Fan G, Yong WS, Madhukumar P, Ooi WS, Chay WY, Dent RA, et al. Minimal clinically important difference (MCID) for the functional assessment of cancer therapy: cognitive function (FACTcog) in breast cancer patients. J Clin Epidemiol. 2014;67(7):811-20.

15. Mendoza TR, Wang XS, Cleeland CS, Morrissey M, Johnson BA, Wendt JK, Huber SL. The rapid assessment of fatigue severity in cancer patients: use of the Brief Fatigue Inventory. Cancer. 1999;85(5):1186-96.
16. Ke Y, Ng T, Yeo HL, Shwe M, Gan YX, Chan A. Psychometric properties and measurement equivalence of the English and Chinese versions of the Beck Anxiety Inventory in patients with breast cancer. Support Care Cancer. 2017; 25(2):633-43.

17. Beck AT, Steer RA, Brown GK. Manual for the Beck depression inventory-II. San Antonio: Psychol. Corp; 1996.

18. Cheung YT, Ng T, Shwe M, Ho HK, Foo KM, Cham MT, Lee JA, Fan G, Tan YP, Yong WS, et al. Association of proinflammatory cytokines and chemotherapy-associated cognitive impairment in breast cancer patients: a multi-centered, prospective, cohort study. Ann Oncol. 2015;26(7):1446-51.

19. Nettiksimmons J, Simonsick EM, Harris T, Satterfield S, Rosano C, Yaffe K. The associations between serum brain-derived neurotrophic factor, potential confounders, and cognitive decline: a longitudinal study. PLoS One. 2014; 9(3):e91339.

20. Driscoll I, Martin B, An Y, Maudsley S, Ferrucci L, Mattson MP, Resnick SM. Plasma BDNF is associated with age-related white matter atrophy but not with cognitive function in older, non-demented adults. PLoS One. 2012;7(4):e35217.

21. Li G, Peskind ER, Millard SP, Chi P, Sokal I, Yu CE, Bekris LM, Raskind MA, Galasko DR, Montine TJ. Cerebrospinal fluid concentration of brain-derived neurotrophic factor and cognitive function in non-demented subjects. PLoS One. 2009:4(5):e5424.

22. Pan W, Banks WA, Fasold MB, Bluth J, Kastin AJ. Transport of brain-derived neurotrophic factor across the blood-brain barrier. Neuropharmacology. 1998;37(12):1553-61.

23. Laske C, Stransky E, Leyhe T, Eschweiler GW, Maetzler W, Wittorf A, Soekadar S, Richartz E, Koehler N, Bartels M, et al. BDNF serum and CSF concentrations in Alzheimer's disease, normal pressure hydrocephalus and healthy controls. J Psychiatr Res. 2007:41(5):387-94.

24. Klein AB, Williamson R, Santini MA, Clemmensen C, Ettrup A, Rios M, Knudsen GM, Aznar S. Blood BDNF concentrations reflect brain-tissue BDNF levels across species. Int J Neuropsychopharmacol. 2011;14(3):347-53.

25. Garcia KL, Yu G, Nicolini C, Michalski B, Garzon DJ, Chiu VS, Tongiorgi E, Szatmari P, Fahnestock M. Altered balance of proteolytic isoforms of probrain-derived neurotrophic factor in autism. J Neuropathol Exp Neurol. 2012;71(4):289-97.

26. Yang F, Je HS, Ji Y, Nagappan G, Hempstead B, Lu B. Pro-BDNF-induced synaptic depression and retraction at developing neuromuscular synapses. $J$ Cell Biol. 2009:185(4):727-41.

27. Lee R, Kermani P, Teng KK, Hempstead BL. Regulation of cell survival by secreted proneurotrophins. Science (New York, NY). 2001;294(5548):1945-8.

28. Lu B. Pro-region of neurotrophins: role in synaptic modulation. Neuron. 2003;39(5):735-8.

29. Pang PT, Teng HK, Zaitsev E, Woo NT, Sakata K, Zhen S, Teng KK, Yung WH, Hempstead BL, Lu B. Cleavage of proBDNF by tPA/plasmin is essential for long-term hippocampal plasticity. Science (New York, NY). 2004; 306(5695):487-91

30. Teng HK, Teng KK, Lee $\mathrm{R}$, Wright $\mathrm{S}$, Tevar $\mathrm{S}$, Almeida RD, Kermani $\mathrm{P}$, Torkin $\mathrm{R}$ Chen ZY, Lee FS, et al. ProBDNF induces neuronal apoptosis via activation of a receptor complex of p75NTR and sortilin. J Neurosci. 2005;25(22):5455-63.

31. Nagappan G, Zaitsev E, Senatorov W Jr, Yang J, Hempstead BL, Lu B. Control of extracellular cleavage of ProBDNF by high frequency neuronal activity. Proc Natl Acad Sci U S A. 2009;106(4):1267-72.

32. Wefel JS, Vardy J, Ahles T, Schagen SB. International Cognition and Cancer Task Force recommendations to harmonise studies of cognitive function in patients with cancer. Lancet Oncol. 2011;12(7):703-8. 